\begin{tabular}{|c|c|c|}
\hline 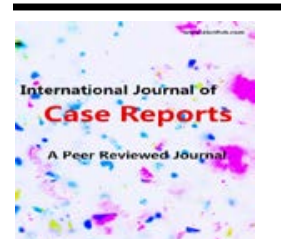 & $\begin{array}{l}\text { International Journal of Case Reports } \\
\text { (ISSN:2572-8776) }\end{array}$ & 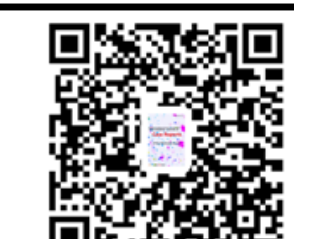 \\
\hline
\end{tabular}

\title{
Foreign Body Aspiration in Adult, What Would You Expect? Case Report
}

\section{Zeyad Faoor Alrais1, Mohamed Ibrahim Shoaib1, Hesham Mohamed ElKholy1, Asad Alsabbah ${ }^{2}$}

${ }^{1}$ Department of Intensive Care Medicine, Rashid Hospital, Dubai Health Authority, Dubai, United Arab Emirates. ${ }^{2}$ Mediclinic City Hospital , Dubai, United Arab Emirates.

\section{ABSTRACT}

Foreign body (FB) aspiration is an uncommon but potentially life-threatening event, while the majority of accidental aspiration events occur in children, adults represent up to $25 \%$ of cases.

When the diagnosis is not established immediately, retained FBs may lead to recurrent pneumonias, bronchiectasis, recurrent hemoptysis, pneumothorax, lung abscesses, pneumo-mediastinum, or other complications. Extraction of aspirated FBs should be undertaken as soon as possible to alleviate acute symptoms and prevent long term complications.

FB aspiration is slightly more common in males. The vast majority of adult patients with FB aspiration have obvious risk factors for aspiration including neurological deficits with swallowing difficulties or altered mental status, neuromuscular disease, intoxication, or have an iatrogenic cause. Still, $10 \%$ of adult patients with FB aspiration have no known risk factors.

Our case is $35 \mathrm{y}$ old male patient who was brought to Accident and Emergency department after being found unresponsive in the street, on arrival to hospital his GCS was $9 / 15$ with pin points pupils , spontaneous breathing and hemodynamic stable. CT brain was unremarkable Patient failed to respond to repeated doses of naloxone and his GCS dropped with episode of apnea so was intubated and mechanically ventilated.

Follow up chest $x$-ray show opacification of the right lung, and ABG reveled hypoxia with respiratory acidosis in spite high ventilator settings, So CT chest was the best option which reveled bronchial obstruction mostly due to foreign body.

Urgent bronchoscopy was done and unexpectedly obstruction was due to chewing gum pieces, aspiration of four large pieces of chewing gum, following that patient remained in the ICU for 2 days where chest $\mathrm{x}$-ray show significant improvement and he was weaned and extubated successfully.
Keywords: Foreign body aspiration, Chewing gum aspiration , bronchoscopy.

*Correspondence to Author:

Dr. Zeyad Alrais

Consultant ICU and Head of Intensive Care Section, Rashid Hospital , Dubai, United Arab Emirates.

How to cite this article:

Zeyad Faoor Alrais, Mohamed Ibrahim Shoaib, Hesham Mohamed ElKholy, Asad Alsabbah. Foreign Body Aspiration in Adult, What Would You Expect? Case Report. International Journal of Case Reports, 2019 4:101

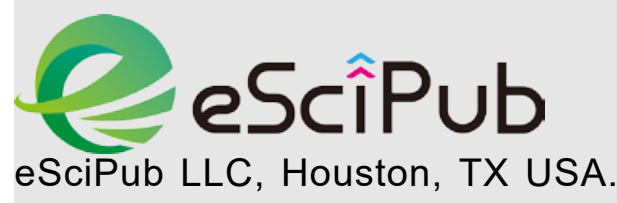

Website: https://escipub.com/ 


\section{Introduction}

Foreign body aspiration (FBA), defined as the introduction of a large particulate material into the tracheobronchial tree.

Foreign body aspiration is an uncommon clinical entity in adults. Children account for the vast majority of all foreign body aspirations reported. Foreign bodies can either be organic (eg, peanuts, peas) or inorganic (eg, plastic caps, pins, screws, nails, teeth).

Clinically, patients may present either with acute respiratory failure requiring urgent intervention or with recent onset of respiratory symptoms, including breathlessness, wheezing, coughing, and expectoration. Identification of foreign body aspiration requires a high index of clinical suspicion, especially in those presenting without a history of aspiration.

FB aspiration is slightly more common in males. The vast majority of adult patients with FB aspiration have obvious risk factors for aspiration including neurological deficits with swallowing difficulties or altered mental status, neuromuscular disease, intoxication, or have an iatrogenic cause. Still, $10 \%$ of adult patients with $\mathrm{FB}$ aspiration have no known risk factors.

The majority of FBs become lodged in the right bronchial tree, favoring the bronchus intermedius and the basal segments of the right lower lobe, owing to a more vertical course of the right main stem bronchus.

Standard postero-anterior and lateral chest radiographs should be obtained in all patients in whom FB aspiration is suspected. Radiographs directly identify the FB in $25 \%$ of patients, as only a minority of FBs such as coins, nails, teeth, or dental appliances are radiopaque. Most FBs are organic and radiolucent (such as food), and therefore not directly visible on chest radiograph.

Chest computed tomography (CT) is more sensitive for identification of FBs, helps with procedural planning, and has become the gold standard of imaging studies when a FB aspiration is suspected.
Flexible bronchoscopy broadened the scope of bronchoscopic interventions to the peripheral airway, allowing removal of foreign bodies lodged more distally with a large variety of dedicated flexible instruments. As technology advanced, technique spread, and practitioners became more experienced, flexible bronchoscopy gradually supplanted rigid bronchoscopy as the most commonly used technique in adults.

Chewing gum aspiration can present a particular dilemma, in that fragments of gum may not be easily extracted. Here we present the clinical and therapeutic challenges that arose in unexpected one episode of chewing gum aspiration.

\section{Case Report}

Our case is $35 \mathrm{y}$ old male patient who was brought to Accident and Emergency department after being found unresponsive in the street, on arrival to hospital his GCS was 9/15 with pin points pupils bilaterally, spontaneous breathing and hemodynamic stable. CT brain was unremarkable.

Patient failed to respond to repeated doses of naloxone and his GCS dropped with episode of apnea so was intubated and mechanically ventilated.

Follow up chest $x$-ray show opacification of the right lung, and $A B G$ reveled hypoxia with respiratory acidosis in spite high ventilator settings, So CT chest was the best option which reveled complete collapse of right lung with an obstructing foreign body was strongly suggested in bronchus intermedius.

Urgent bronchoscopy was done and unexpectedly obstruction was due to chewing gum pieces, aspiration of four large pieces of chewing gum, following that patient remained in the ICU for 2 days where chest $x$-ray show significant improvement and he was weaned and extubated successfully.

\section{Discussion}

Tracheobronchial foreign body (FB) aspiration is an uncommon but potentially life-threatening 
Zeyad Faoor Alrais et al., IJCR, 2019 4:101

event in adults (1). Clinically, patients may present either with acute respiratory failure requiring urgent intervention or with recent onset of respiratory symptoms, including breathlessness, wheezing, coughing, and expectoration. Identification of foreign body aspiration requires a high index of clinical suspicion, especially in those presenting without a history of aspiration ${ }^{(2,3)}$. FB aspiration is slightly more common in males, many adult subjects with tracheobronchial foreign bodies described in previous studies had an underlying risk factor, such as neuromuscular disease, head trauma, alcohol intoxication, or altered sensorium ${ }^{(2,4)}$. Our presented case had altered sensorium with low GCS which was the predisposing factor of his aspiration.

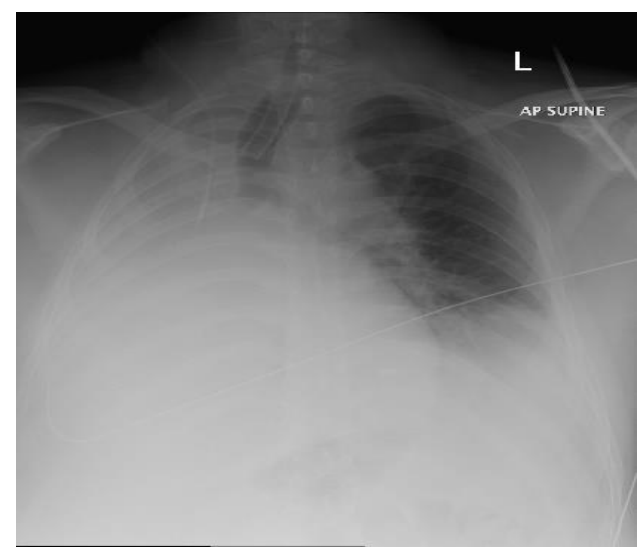

$1^{\text {st }}$ chest $X$ ray show right lung Opacity

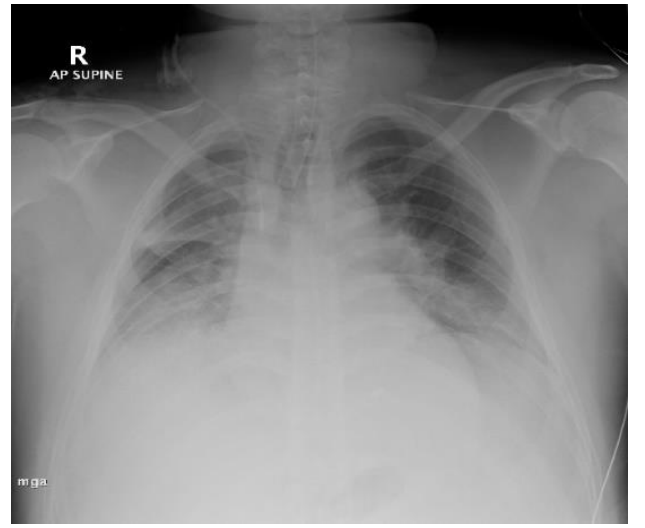

chest $X$ ray post bronchoscopy

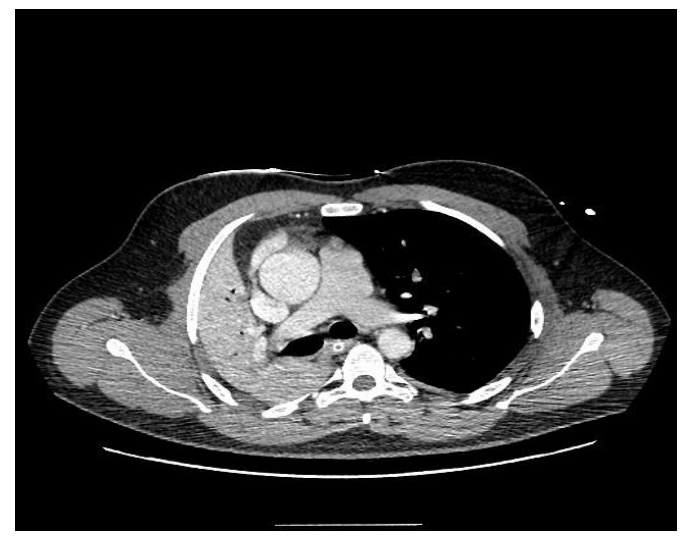

CT Findings(1)

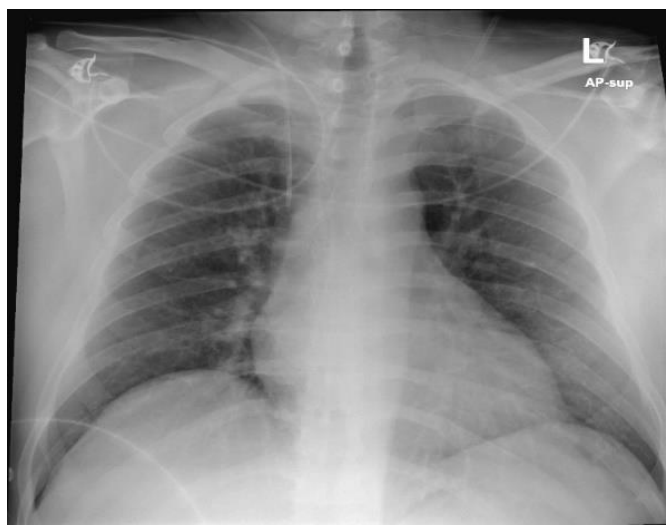

chest $X$ ray on discharge

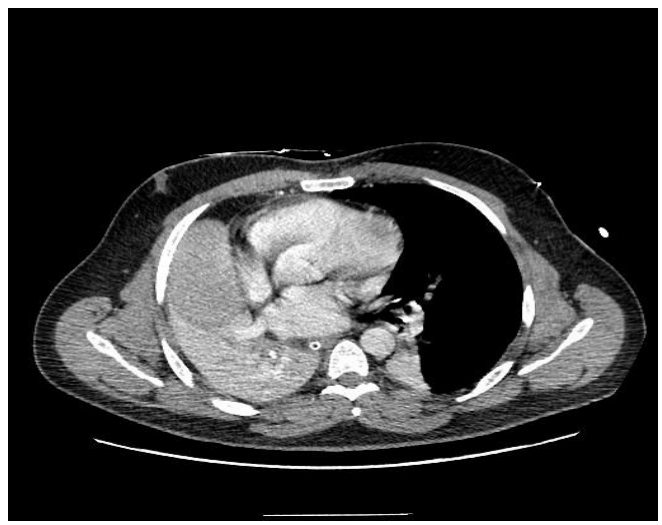

CT Findings (2) 


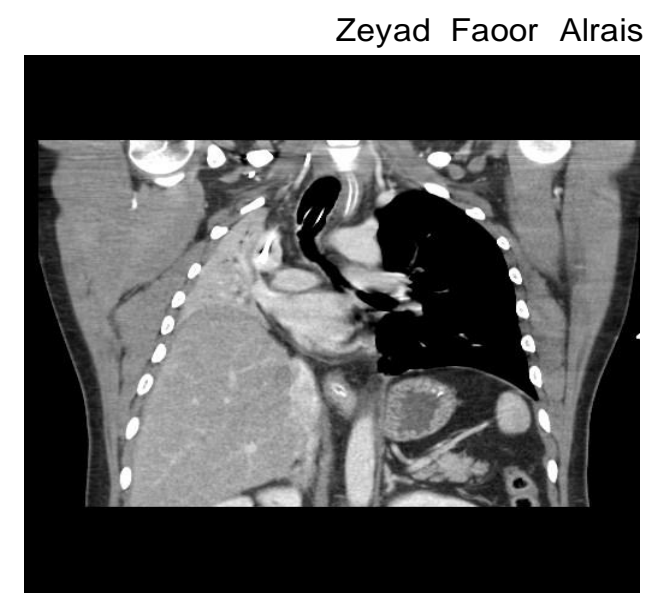

CT Finding (3)

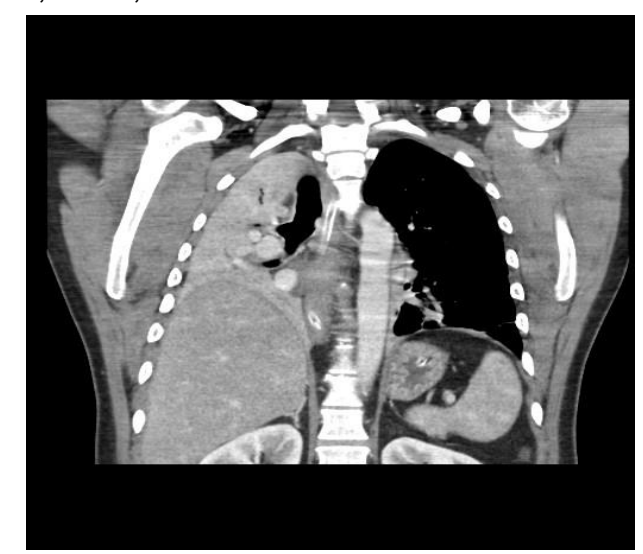

CT Finding (4)

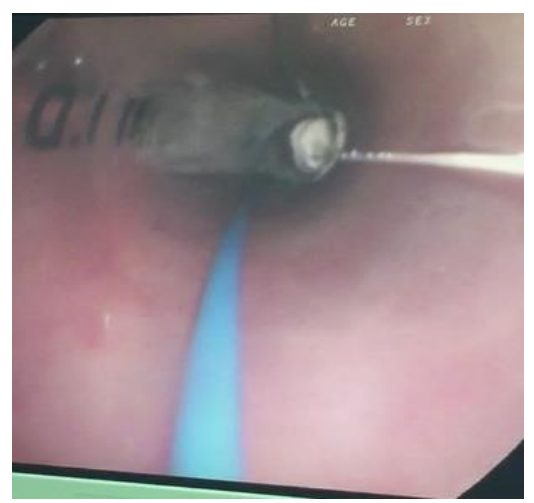

Bronchoscopic extraction (1)

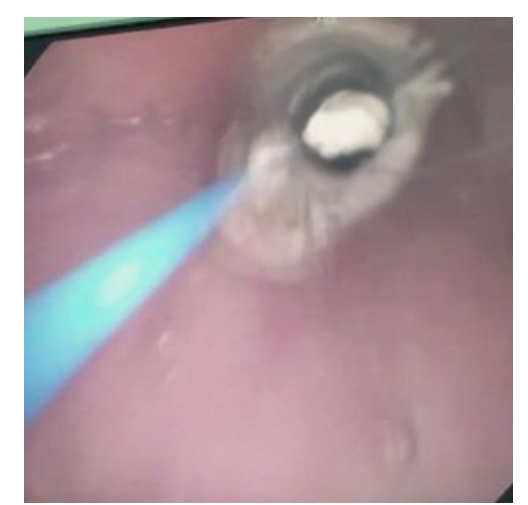

Bronchoscopic extraction (2)

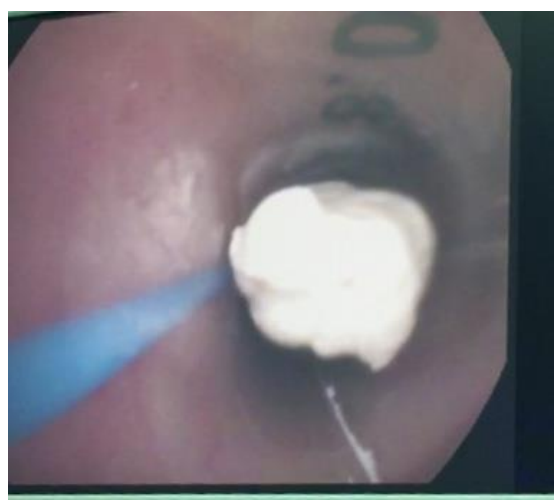

Bronchoscopic extraction (3)

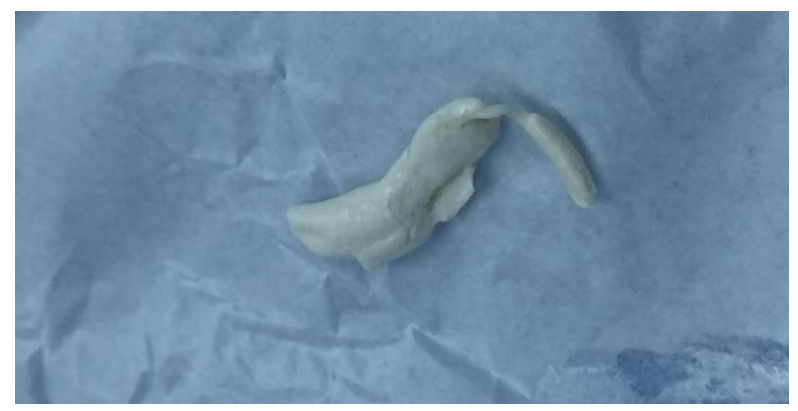

Extracted Chewing gum piece

The majority of FBs become lodged in the right bronchial tree, favoring the bronchus intermedius and the basal segments of the right lower lobe, owing to a more vertical course of the right mains stem bronchus ${ }^{(1,5,6)}$, and our presented case showed clearly the same finding of right bronchus intermedius location of the FB.

Standard postero-anterior and lateral chest radiographs should be obtained in all patients in whom FB aspiration is suspected. Radiographs directly identify the $\mathrm{FB}$ in $25 \%$ of patients, as

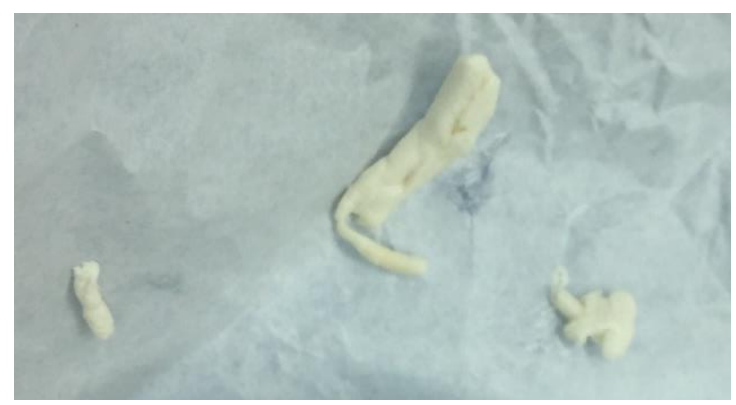

Extracted pieces of chewing gum

only a minority of FBs such as coins, nails, teeth, or dental appliances are radiopaque $(1,6)$. Most FBs are organic and radiolucent (such as food), and therefore not directly visible on chest radiograph (2.7).

Chest radiographs can demonstrate indirect, non-specific, findings of atelectasis, hyperinflation, bronchiectasis, or lobar consolidation in the majority of patients. Expiratory films can assess for focal hyperinflation related to ball-valve phenomena caused by a FB that is partially obstructing an 
airway on inhalation and completely obstructing an airway on expiration. In $14-35 \%$ of patients the chest radiograph will be entirely normal $1,2,7)$

Chest computed tomography is more sensitive for identification of FBs, helps with procedural planning, and has become the gold standard of imaging studies when a FB aspiration is suspected, In our case the CT chest was done urgently for the high clinical suspicion which allowed for the rapid recognition of the aspirated FB ${ }^{(8)}$.

Flexible bronchoscopy has, in general, supplanted rigid bronchoscopy as the initial procedure for evaluation and management of FB aspiration. Flexible bronchoscopy allows for a more comprehensive airway survey and has an overall $90 \%$ success rate for $\mathrm{FB}$ removal $(9,10)$

Chewing gum aspiration can present a particular dilemma, in that fragments of gum may not be easily extracted, extraction of the aspirated chewing gum was challenging in the presented case as the pieces were fragile and sticky, the extracted 4 pieces were completely obstructing the bronchi (11).

\section{Conclusion}

This case illustrated un expected presentation of chewing gum aspiration in an adult causing complete collapse of the lung lobes.

\section{Conflict of Interest}

The author(s) declared no potential conflicts of interest with respect to the research, authorship, and/or publication of this article.

\section{References}

1. J Hewlett, O Rickman, R Lentz, et al. Foreign body aspiration in adult airways: therapeutic approach. J Thorac Dis. 2017 Sep; 9(9): 33983409.

2. Sehgal IS, Dhooria S, Ram B, et al. Foreign Body Inhalation in the Adult Population: Experience of 25,998 Bronchoscopies and Systematic Review of the Literature. Respir Care 2015;60:1438-48.

3. Rafanan AL, Mehta AC. Adult airway foreign body removal. What's new? Clin Chest Med 22(2):319-330, 2001.

4. Mise K, Jurcev Savicevic A, Pavlov N, Jankovic $S$. Removal of tracheobronchial foreign bodies in adults using flexible bronchoscopy: experience 1995-2006. Surg Endosc 2009;23(6):1360-1364.

5. Lin L, Lv L, Wang Y, et al. The clinical features of foreign body aspiration into the lower airway in geriatric patients. Clin Interv Aging 2014;9:16138.

6. Blanco Ramos M, Botana-Rial M, García-Fontán $E$, et al. Update in the extraction of airway foreign bodies in adults. $J$ Thorac Dis 2016;8:3452-6.

7. Boyd M, Chatterjee A, Chiles $C$, et al. Tracheobronchial foreign body aspiration in adults. South Med J 2009;102:171-4.

8. Rodrigues AJ, Oliveira EQ, Scordamaglio PR, et al. Flexible bronchoscopy as the first-choice method of removing foreign bodies from the airways of adults. J Bras Pneumol 2012;38:31520.

9. Dong YC, Zhou GW, Bai C, et al. Removal of tracheobronchial foreign bodies in adults using a flexible bronchoscope: experience with 200 cases in China. Intern Med 2012;51:2515-9.

10. Casalini AG, Majori M, Anghinolfi M, Burlone E, D'Ippolito R, Toschi $M$, et al. Foreign body aspiration in adults and in children: advantages and consequences of a dedicated protocol in our 30-year experience. J Bronchology Interv Pulmonol 2013;20(4):313-321.

11. Matthew F. Abts, Katherine Hicks, Dana Thompson, Lauren et al. Therapeutic Challenges after Chewing Gum Aspiration in a Toddler: A Case Report .Ann Am Thorac Soc. 2016 Jun;13(6):988-90.

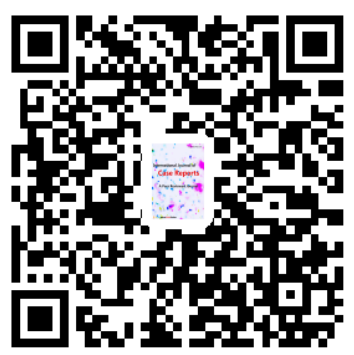

